\title{
Las estrategias de especialización inteligente: una estrategia territorial para las regiones
}

\author{
Smart specialization strategies: \\ a territorial strategy for regions
}

\author{
Mikel Navarro Arancegui ${ }^{1}$ \\ Mari José Aranguren Querejeta ${ }^{1}$ \\ Edurne Magro Montero ${ }^{2}$ \\ Orkestra-Instituto Vasco de Competitividad y Deusto Business School (España)
}

Recibido el 14 de septiembre de 2011, aceptado el 18 de enero de 2012

$\mathrm{N}^{\mathrm{o}}$ de clasificación JEL: O21, O38, R11, R58

DOI: $10.5295 / \mathrm{cdg} .110310 \mathrm{mn}$

\section{Resumen:}

A pesar de que existe un amplio reconocimiento de que los territorios necesitan desarrollar estrategias de innovación para la construcción de ventajas competitivas, hasta ahora no han sido explicitados el contenido y las particularidades del término estrategia aplicado al ámbito territorial, por lo que se ha utilizado frecuentemente con significados ambiguos. Este artículo trata, en primer lugar, de de esclarecer qué contenidos debería presentar una estrategia territorial, así como las características de su proceso de formación. Además, se presenta y analiza un tipo de estrategia territorial que actualmente está siendo particularmente difundida por la Comisión Europea para su implementación en el ámbito regional: la estrategia de especialización inteligente. Este artículo pretende además analizar los aspectos discutibles o que permanecen abiertos a un mayor desarrollo y precisión en torno a este concepto.

Palabras clave:

Estrategia, territorio, especialización inteligente, innovación.

\begin{abstract}
:
There is clear acknowledgment in the literature of the importance of developing regional innovation strategies to build to build competitiveness advantages. However the concept remains fuzzy in terms of its limits and contents applied to the territorial field. This paper aims to shed light on the contents a territorial strategy should include as well as on the main characteristics characteristics that present the development process of territorial strategies. Moreover, this paper analyses one concrete type of territorial strategy, which is currently being disseminated by the European Commission for regional development: the smart specialization strategy. In consequence, this paper analyses the aspects that remain under discussion and opened to a greater development and precision under the smart specialization concept.
\end{abstract}

Keywords:

Strategy, territory, smart specialization, innovation.

${ }_{1}^{1}$ Orkestra-Instituto Vasco de Competitividad y Deusto Business School.
${ }^{2}$ Orkestra-Instituto Vasco de Competitividad 


\section{INTRODUCCIÓN}

Dentro de las diferentes corrientes que desde comienzos de los 90 surgen para analizar la estrecha relación que competitividad e innovación guardan con el desarrollo (Porter, sistemas de innovación, modelo de Triple Hélice...) hay un progresivo explícito reconocimiento de que los territorios necesitan desarrollar estrategias de innovación para la construcción de ventajas competitivas, a partir de su particular configuración de recursos, competencias y capacidades y de las tendencias existentes en el entorno (Porter, 2010; Asheim et al. 2006; Etzkowitz y Klofsten, 2003). Hoy es habitual encontrar este término tanto en la literatura de desarrollo económico y de la innovación como en los discursos de los decisores públicos.

Sin embargo, a semejanza de lo acontecido con términos como competitividad, benchmarking, etc. que se han importado de otros campos al ámbito económico y territorial (Navarro et al., 2001), ese nuevo uso no ha ido precedido por una explicitación de su contenido y de las peculiaridades que tal término debe adoptar en esos nuevos ámbitos; y ello ha dado lugar a que se haya utilizado frecuentemente con significados ambiguos o incluso contradictorios. El primer apartado de este artículo tiene, por consiguiente, exponer qué contenido debe contemplar la estrategia cuando aparece referida al territorio, así como qué características debe presentar el proceso de formación de la estrategia en ese ámbito.

Tras esa primera reflexión, en un segundo apartado se analiza el tipo de estrategia territorial que, diseñada inicialmente por Dominique Foray con la colaboración de diversos autores (Foray y Van Ark, 2007; Foray et al., 2009; Foray, 2009a; Foray, 2009b; David et al., 2011; McCann y Ortega-Argilés, 2011), ha sido recogida en una serie de documentos y comunicaciones de la Comisión Europea (Informe Barca, 2009; COM(2010) 553 final; SEC(2010) 1183): las estrategias de especialización inteligente. Este tipo de estrategias van a tener una gran relevancia en el futuro desarrollo regional europeo, pues por ellas van a ir las recomendaciones y el reparto de fondos que para el desarrollo regional se realicen desde las instancias europeas. Sin embargo, como suele ser habitual en las primeras formulaciones de toda aproximación de este tipo, hay determinados aspectos de las mismas que resultan discutibles, o necesitados de precisión o adicional desarrollo, tal como se mostrará.

Para finalizar, en el último apartado, se extraen una serie de conclusiones y recomendaciones para el enriquecimiento y aplicación de las estrategias territoriales y, concretamente, las de especialización inteligente.

\section{LA ESTRATEGIA TERRITORIAL}

Si bien no hay consenso sobre qué debe entenderse por estrategia en el ámbito empresarial, cabría ver de modo general la estrategia como una idea unificadora que liga acción y objetivo, es decir, una línea de actuaciones para la consecución de los objetivos de una organización (White, 2004). Un rasgo que añade Porter (1980 y 1985) a esa aproximación general a la idea de estrategia es que con ella la empresa debe buscar un posicionamiento diferenciado, idea que incluso será más reforzada por la corriente de los recursos y capa- 
cidades. En particular, Porter (1996) señala que "estrategia es la creación de una posición única y valiosa, que implica un conjunto de actividades diferenciadas” (p. 53).

Las estrategias empresariales y territoriales, aunque comparten el uso de un mismo sustantivo (a saber, el término estrategia), son diferentes, por lo menos en tres planos: (i) en los objetivos, (ii) en las preguntas básicas para definir el posicionamiento estratégico y las bases que los sustentan, y (iii) en su proceso de formación. O dicho de modo más sintético: en el para qué, en el qué, y en el cómo/por quién.

Primero, en cuanto a los objetivos, la corriente mayoritaria en el mundo anglosajón propugna que la estrategia empresarial debe buscar maximizar su valor y la rentabilidad económica (Thompson et al., 2008), aunque hay planteamientos que amplían el foco y reclaman que se tomen en cuenta los intereses de todos los grupos de interés o stakeholders, e incluso objetivos éticos y sociales (White, 2004). En cuanto a los territorios, en general se considera que la estrategia de desarrollo lo que debe buscar es mejorar el bienestar de la población, ligando éste a la consecución de objetivos económicos (visión restrictiva), o también a objetivos sociales y medioambientales (visión amplia) ${ }^{1}$.

En segundo lugar, en la empresa, se busca una diferenciación tanto con respecto a los mercados (es decir, qué tipo de productos produce la empresa, a qué clientes y necesidades atiende, y qué acceso o a qué mercados), como a los recursos y capacidades internos de la empresa. En las regiones, en cambio, la diferenciación pasa por decidir en qué actividades destacarán, qué activos específicos se ofrecerán para hacer que las empresas opten por localizarse en ellas, en qué actores descansarán tales actividades y cuál será el rol o la conexión de la región con respecto a otros territorios (sean éstos las regiones vecinas y el resto del Estado, la región geo-económica en que se ubica o la economía mundial) y de los diferentes espacios que la componen.

En tercer lugar, en cuanto al proceso de formación de la estrategia, la literatura de Dirección de Empresas presenta una gran diversidad de planteamientos, desde los que siguen considerando que ella es función fundamentalmente de los directivos (Thompson et al., 2008), hasta los que plantean procesos más abiertos en los que "prácticamente cualquiera de la organización puede resultar ser un estratega" (Mintzberg 1994; p. 26). Pero en todo caso, la estrategia es algo que obliga a todos los componentes de la organización, y en tal sentido tiene un componente coercitivo. En los territorios, en cambio, los procesos participativos son esenciales, ya que ninguno de los múltiples actores y administraciones dispone de la autoridad, el conocimiento o los recursos para imponer o llevar adelante unilateralmente la estrategia para el territorio por él deseada.

\subsection{Actividades económicas, activos, actores y posicionamiento externo-interno}

Como más arriba se ha señalado, la diferenciación o establecimiento de una proposición única de valor del territorio pasa, en lo referente al contenido, por apostar por un conjunto de actividades en que destacar, por unos activos específicos en que descanse el

\footnotetext{
${ }^{1}$ Desde una perspectiva más integral, resulta sugerente el instrumento propuesto por McCann (2011) para la identificación de los principales rasgos de una región en las tres dimensiones de la Estrategia de Lisboa, para permitir a los decisores públicos un mejor diseño de estrategias de desarrollo integradas.
} 
atractivo del territorio en esas actividades, por el tipo de actores que desarrollarán ese tipo de actividades y por el modo de conexión de la región con respecto al exterior y de estructura del territorio cara al interior. No obstante, conviene que previamente a ese posicionamiento, el territorio identifique los grandes desafíos que para él se derivan del estadio de desarrollo en que se encuentre. Efectivamente, si bien resultan acertadas las críticas que, autores como Rodríguez-Pose (2011) efectúan a los que propugnan modelos de desarrollo lineales semejantes a los de Rostow (1960), dado que en última instancia implican que los territorios deben proseguir políticas de desarrollo iguales que no toman en cuenta la combinación de factores temporales y espaciales de cada caso, sí que hay algunos retos comunes para los territorios derivados del estadio de desarrollo en que se encuentran y que condicionan el marco desde el que su diferenciación o establecimiento de una proposición única de valor debe llevarse a cabo.

Porter (1990) y la OECD (2011) han planteado, desde diferentes bases (uno más basado en una reflexión sobre un amplio número de casos; y la otra a partir de una tipología regional realizada a partir de 12 indicadores ligados a desempeño, mercado de trabajo e innovación tecnológica), una división en tres grandes tipos de desafíos. Según Porter, los territorios atraviesan tres grandes estadios de desarrollo competitivo, en cada uno de los cuales los grandes retos establecen el marco general en el que establecer las estrategias territoriales: en el primero, el nivel de desarrollo del territorio es bajo y fundamentalmente se compite en factores, con estrategias en las que se compite en base a bajo coste; el segundo sería un estadio de desarrollo intermedio, en el que se compite más basándose en la inversión y en la eficiencia; y el tercero sería el de los territorios más desarrollados, que compiten basándose en la innovación ${ }^{2}$. En cuanto a la OECD (2011), los tres grandes tipos de elecciones y familias de estrategias que afrontan las regiones son: el catching-up, el apoyo a la transformación socio-económica y la construcción sobre las existentes ventajas científico-tecnológicas. Aunque la caracterización que la OECD hace de las regiones que deberían dar prioridad a una u otra de estas estrategias es más compleja de lo que aquí podemos permitirnos reproducir (véanse, especialmente, páginas 78-91 de su informe), simplificando cabría emparejar los territorios que deben perseguir estrategias de catchingup con los que, en la categorización porteriana, compiten en factores; los que persiguen el apoyo a la transformación socio-económica con los que compiten en el estadio de la eficiencia y la inversión; y aquellos para los que se les recomienda la construcción sobre las existentes ventajas científico-tecnológicas, con los que compiten en el estadio de la innovación.

Centrándonos en el tipo de actividades por las que apueste un territorio, un primer dilema estratégico que se plantea es el de qué tipo de economías de aglomeración perseguirá explotar el territorio: de localización, impulsando la especialización sectorial; de urbanización, impulsando el mero tamaño y diversificación; o de variedad relacionada. La reciente literatura empírica parece apoyar la idea de que es este último tipo de aglomeración el que ofrece mejores resultados económicos (Frenken et al., 2007; Boschma y Iammarino, 2009; Nefke et al., 2011; Boschma et al., 2012). En todo caso, cuando se plantea esa necesidad de optar por un conjunto de actividades, lo que se reconoce cada vez por un número mayor

\footnotetext{
${ }^{2}$ Esta división de los territorios en tres grandes estadios de desarrollo competitivo ha sido asumida, entre otros, por el conocido informe The Global Competitiveness Report que publica el World Economic Forum.
} 
de analistas, es que el imparable desarrollo de "redes de producción globales" y la pareja fragmentación o desverticalización del proceso productivo hace que no quepa plantear o aspirar a que tales actividades correspondan a sectores o clústeres completos, sino sólo a determinados conjuntos de actividades (Breznitz, 2007), que con frecuencia tienden a estar relacionadas por compartir un conjunto de habilidades o bases de conocimiento (Henning et al., 2010).

Un segundo dilema, muy ligado al anterior, radica en impulsar lo que la literatura denomina el deepening (es decir, la profundización y especialización en aquellas actividades en que tiene ventajas), o impulsar la capacidad de shifting o de adaptación a los cambios y reinvención de uno mismo (Pontikakis et al., 2009). Etzkowitz y Klofsten (2003) plantean este dilema de forma algo diferente, cuando dicen que algunos territorios persiguen ser una learning region, que busca construir sobre activos existentes, con procesos de innovación incremental; mientras que otros persiguen ser una innovating region, que busca crear nuevos activos, ligados a una base de investigación. En general, ante los riesgos de lock-in que la primera implica, la literatura se inclina por impulsar la diversidad (véase, por ejemplo, Freeman 1997; Dalum et al., 1992; Carlsson, 1994).

En cuanto a los activos por los que apueste el territorio, dos son asimismo los grandes dilemas a que se enfrenta la estrategia territorial: (i) el centrarse en el desarrollo de unas core capacidades, o el buscar un balance más equilibrado de los diferentes tipos de activos; y (ii) el trabajar con una visión más amplia o más restrictiva de la innovación y sus determinantes. Respecto al primer dilema, autores de muy diferentes corrientes sostienen que la corrección de los puntos débiles y la combinación de diferentes modos de aprendizaje, innovación o bases de conocimiento ofrece mejores resultados que el concentrarse en desarrollar al máximo los puntos fuertes o el desarrollo de estrategias basadas en un único factor (Hansen y Birkinshaw, 2007; Soete, 2006; Arundel y Hollanders, 2007; Lundvall y Lorenz, 2007; Jensen et al., 2007; Asheim, 2009; Frenz y Lambert, 2009; Mairesse y Mohnen, 2007; Criscuolo et al., 2011; Wintges y Hollanders, 2010). En cuanto al segundo dilema, frente a planteamientos que reducen los componentes del sistema a aquellos ligados con las actividades de I+D (por ejemplo, Nelson, 1993; Niosi, 2010; Etzkowitz y Leydesdorff, 2000), tendríamos los enfoques más amplios que tratan de incluir en el análisis todos los componentes y relaciones del sistema que inciden significativamente en la innovación (Lundvall, 1992; Edquist, 1997; Asheim y Gertler, 2005). Un enfoque restrictivo del sistema de innovación, muy centrado en la $\mathrm{I}+\mathrm{D}$, puede facilitar el análisis y ajustarse bien a las características de un territorio muy avanzado en ciencia (por ejemplo, Cambridge); pero, en contrapartida, deja sin respuesta a la mayor parte de la actividad económica que tiene lugar en territorios menos avanzados, cuya innovación está basada en otro tipo de actividades.

En tercer lugar, tanto las primeras tipologías o categorizaciones desarrolladas por los padres de la literatura de los sistemas regionales de innovación (Cooke, 1998; Asheim e Isaksen, 1997 y 2002) como los análisis sobre las razones que explican el éxito de determinados países emergentes (Breznitz, 2007), destacan que un rasgo fundamental de sus estrategias de desarrollo radica en su posicionamiento con respecto al tipo de empresa que se primaba (grandes campeones nacionales versus pymes; empresas y organizaciones privadas versus un modelo mixto con una mayor presencia del sector público). 
Por último, el territorio tiene que posicionarse tanto hacia fuera como hacia dentro. Con el posicionamiento hacia fuera hacemos referencia a qué tipo de combinación de factores externos e internos aspira, tanto en términos de conocimiento como de otros factores productivos. Frente a una tendencia a hacer descansar, de modo casi exclusivo, el desarrollo económico y la innovación en factores y relaciones internas del sistema, fueron múltiples los analistas que advertían de la imposibilidad, ineficiencia y riesgo de lock-in por depender exclusivamente del conocimiento generado dentro del sistema (Kaufmann y Tödtling, 2000; Archibugi et al., 1999) y las primeras tipologías conceptuales de los sistemas regionales de innovación tomaban tal conexión con el exterior como un factor fundamental de la caracterización del territorio (Cooke, 1998; Asheim e Isaksen, 1997 y 2002). Ello ha dado lugar a la ya conocida propuesta de "local buzz and global pipelines", formulada por Bathelt et al. (2003). Como indica Breznitz (2007), las estrategias territoriales difieren en función del peso que se le asigna a las multinacionales (que construyen, gestionan y en buena medida controlan las "redes de producción globales") y a la financiación por los mercados globales. Pueden diferir notablemente los output que las multinacionales persiguen obtener de las empresas del territorio, así como las actividades que ellas desarrollan en tales territorios (desde el mero ensamblaje, pasando por la producción manufacturera o la realización de sofisticadas actividades de I+D).

El posicionamiento hacia adentro haría referencia a la necesidad de no tomar el territorio o la región como algo homogéneo, sino compuesto por diferentes places o espacios con características diferenciadas (Bathelt, 2003; MacKinnon et al., 2002; Muscio, 2006; Uyarra, 2007; Uyarra y Flanagan, 2009; McCann, 2011). La estrategia territorial debe perseguir, en consecuencia, un modo de inserción y relación determinada con los restantes territorios, buscando la combinación más apropiada de conocimientos externo e interno (que se encuentra ligada a la capacidad de absorción de la región); así como qué configuración y rol se persigue para las diferentes zonas constituyentes del territorio.

\subsection{El proceso de formación de la estrategia}

Aunque el contenido o sustancia (el "qué") y el proceso de formación (el "cómo" y "por quién") de la estrategia se condicionan mutuamente, suele ser habitual distinguirlos a efectos de exposición (Hax y Majluf, 1996). De las múltiples dimensiones que pueden ser consideradas en el proceso de formación de la estrategia, por la particular relevancia que presentan para el territorio y las limitaciones de espacio nos centraremos solamente en dos: en su carácter deliberado o emergente; y en el tipo de liderazgo y gobernanza que requieren.

Mintzberg (1994) introdujo una interesante distinción entre lo que él denomina estrategias deliberadas ("deliberate strategies") y estrategias emergentes ("emergent strategies"), posteriormente retomada e importada para el análisis territorial por Sotarauta (2004). Mintzberg sostiene que, paralelamente a las actuaciones que se adoptan en respuesta a un plan predeterminado, hay cantidad de decisiones que se adoptan como respuesta emergente a circunstancias no previstas, y que, cuando hay todo un conjunto de actuaciones así llevadas a cabo que convergen en el tiempo y presentan una consistencia o patrón de comportamiento, constituirían una estrategia emergente ("emergent strate- 
gy"). Dado que tanto la deliberada como la emergente son estrategias, Mintzberg prefiere hablar de proceso de formación de la estrategia, en lugar de proceso de formulación de la estrategia.

Las estrategias deliberadas responderían a una aproximación clásica a la estrategia, en la que la planificación estratégica racional (deliberada) constituiría el proceso clave. Habitualmente, esta suele ser la aproximación imperante en las estrategias territoriales, de modo que por estrategia territorial suele normalmente entenderse: "el resultado de un proceso consciente, que se plasma en un documento escrito en un 'programa/plan de desarrollo regional' o en una 'estrategia de desarrollo regional"' (Sotarauta, 2004; p. 19). Son muchos los defectos que suele presentar esta aproximación clásica (véase, para mayor detalle, Sotarauta, 2004), pero quizá el más destacable es la separación entre las fases de diseño e implementación, de modo que finalmente los planes suelen ser "de todos y de nadie" y suelen quedarse en el papel.

Las estrategias emergentes responderían, en cambio, a una aproximación procesual a la estrategia. La estrategia consistiría en un proceso "en el que diferentes objetivos y estrategias de múltiples agentes de la región se concilian, varios intereses se equilibran, y se buscan y coordinan continuamente puntos de encuentro y recursos concretos entre múltiples objetivos" (Sotarauta, 2004; p. 24). En esta aproximación, la estrategia no precede a la acción, sino que emerge simultáneamente con esta y se reinventa continuamente, de modo que las estrategias de las diferentes organizaciones se van haciendo cada vez más paralelas a través del encuentro y el diálogo. Pero esta aproximación presenta también riesgos, porque sin ejercicios de prospectiva y tener en cuenta el futuro, las ideas estratégicas que espontáneamente surjan es posible que carezcan de visión a largo.

Lo deseable sería una estrategia mixta que combinara las ventajas de ambas aproximaciones, y evitara sus debilidades. Esto es, una estrategia que incorpore métodos y análisis, de modo que se reflexione sobre elementos clave y posibles escenarios de futuro; pero que contenga espacios para la discusión y negociación (que permitan aprendizajes y visiones compartidas), así como para la emergencia de otros elementos de la estrategia durante su implantación.

Como señalan Nahapied y Ghoshal (1998), para que tenga lugar la colaboración y el proceso de generación de una visión compartida es necesaria la existencia de capital social. Los citados autores distinguen tres dimensiones clave del mismo: existencia de redes y espacios, en que se puedan llevar adelante esos procesos (dimensión estructural); relaciones personales de confianza, amistad,... que influyan en su comportamiento (dimensión relacional); y lenguajes, códigos, interpretaciones... compartidos, en los que hay que ir alcanzando cada vez mayor convergencia (dimensión cognitiva) ${ }^{3}$.

En cuanto al liderazgo y tipo de gobernanza del proceso, cabe señalar que en un contexto complejo, donde el conocimiento necesario para responder a los retos detectados está distribuido no sólo entre distintos agentes del territorio, sino a nivel global, la estrategia regional no puede ser liderada por un solo agente. Se necesita una multiplicidad

\footnotetext{
${ }^{3}$ A una realidad semejante hace en parte referencia Camagni (2011) con su concepto de "identidad territorial": "presencia de 'capital social'; capacidad de desarrollo de visiones compartidas sobre el futuro; know-how y especificidades locales, 'vocaciones' productivas y ventajas competitivas de cada territorio" (p. 61).
} 
de partenariados público-privados, que respondan a los diferentes retos estratégicos que la región tiene y que conviertan al conjunto de participantes diversos de la región en una red de líderes resiliente, capaz de ejecutar y supervisar decisiones y programas de actuación complejos. En cada etapa de desarrollo de la estrategia se necesitan competencias que no siempre un mismo actor (persona u organización) posee, por lo que en diferentes momentos del proceso el rol de cada agente puede variar y puede ser necesario que el liderazgo vaya rotando. Es importante que cada actor sepa entender el momento en que su papel puede hacerse crítico en el proceso y esté dispuesto a asumir responsabilidades.

Cuando el objetivo que se persigue consiste en la consecución de un mejor nivel de bienestar para sus ciudadanos, es vital que las decisiones clave adoptadas por las redes de agentes reflejen adecuadamente el conjunto de intereses de la sociedad (Sugden y Wilson, 2002 y 2005). Los procesos de toma de decisiones (quiénes participan y cómo se articula dicha participación) se convierten en críticos. El proceso de formación de la estrategia requiere una gobernanza o coordinación auto-organizativa (heterarquía) entre distintos agentes (Jessop, 1998). Los agentes que participan en la gobernanza pueden incluir instituciones públicas - por ejemplo, gobiernos de distintos niveles- y también instituciones privadas y personas autónomas en su operativa, pero interdependientes. El hecho de que sea necesaria una aproximación multinivel para poder entender las decisiones que se toman en distintos niveles, dentro y fuera del territorio, hace que el grado de complejidad del concepto aumente. Frente a la gobernanza basada en la jerarquía, la nueva gobernanza basada en redes se caracterizaría por primar el enfoque de procesos sobre el de estructuras, por concebir las fronteras como abiertas y no como cerradas, por impulsar la colaboración más que la coordinación, por descansar en la confianza en lugar de en el rendimiento de cuentas, y por desarrollar el empoderamiento de las distintas comunidades en vez del poder jerárquico (Garmann Johnsen et al., 2008).

Centrándonos más en particular en el papel del Gobierno en este proceso, la participación e implicación de niveles supra-regionales resulta imprescindible no sólo porque sus estrategias y políticas van a afectar de modo decisivo a las de la región (Uyarra y Flanagan, 2009), sino también porque, como señala Barca (2011), la combinación de ese componente exógeno con los endógenos del territorio, además de comportar conocimiento y valores de 'fuera', puede resultar clave para superar los frecuentes fallos que tienen lugar en las élites y gobiernos locales ${ }^{4}$.

Por último, aparte del tema competencial, al que cada vez se le presta mayor atención y del cual comienza a disponerse de mayor información, no podemos olvidarnos de las capacidades de gobierno para hacer uso de tales poderes (Ismeri Europa y Applica, 2010; Walendowski et al., 2011; OECD, 2011). Tal como pone manifiesto Breznitz (2007), tales capacidades están determinadas por el perfil de la burocracia de las Administraciones, es decir, por "los modos en que se construye la burocracia, los orígenes sociales y nivel edu-

\footnotetext{
${ }^{4}$ Barca (2011), refiriéndose a esas élites y gobiernos locales, habla de las trampas o problemas derivados de "la incapacidad (dado que la capacidad es dependiente de la senda anterior), falta de disposición (por su deseo de maximizar su propia cuota en un output dado) o insuficiencia (flujos centrípetos de trabajo y capital debidos a efectos de aglomeración) para proporcionar las apropiadas instituciones e inversión, que hacen necesaria una intervención exógena para promover el cambio endógeno" (p. 47)
} 
cativo de su personal y su capacidad para conformar el proceso de decisión" (p. 32) . El tipo de burocracia existente va a condicionar notablemente la capacidad y el modo en que tenga lugar la relación del Gobierno con el resto de los actores del territorio.

\section{LA ESTRATEGIA DE ESPECIALIZACIÓN INTELIGENTE}

El enfoque de las estrategias de especialización inteligente (que en la literatura con frecuencia aparecen denominadas por su acrónimo inglés: $\mathrm{S}^{3}$, que nosotros también utilizaremos por abreviar) surge de la constatación de que la mayoría de los poderes regionales estaban aplicando enfoques o políticas miméticas unas de otras, sin tomar realmente en cuenta la pluralidad y diversidad de sus contextos. Eso estaba llevando a una excesiva uniformidad, y a que no se alcanzaran en las regiones los umbrales críticos necesarios para una mínima eficiencia y eficacia de las inversiones y para que la UE pudiera jugar un papel líder en la mayoría de las áreas en el mundo (véase documento COM(2010) 553 final). Ante eso, el documento de trabajo del personal de la Comisión Europea sobre la contribución de la política regional al crecimiento inteligente en la Europa 2020 (véase SEC(2010) 1183) propugna que se apliquen en las regiones estrategias de especialización inteligente.

De acuerdo con lo expuesto en el anterior apartado, en el análisis de dichas estrategias cabría distinguir dos planos: el del propio contenido de la estrategia, y el del proceso o modo de llevarla a cabo.

\subsection{Contenido de las estrategias de especialización inteligente.}

En el documento de la Comisión $\operatorname{COM(2010)~} 553$ final antes citado esta estrategia se define de la siguiente manera: "En esencia, la especialización inteligente trata de poner mayor énfasis en la innovación y en concentrar los escasos recursos humanos y financieros de I+D+i en unas pocas áreas competitivas globalmente" (p. 41). Así, frente a planteamientos como los de Gill (2010 y 2011) o el informe World Development Report 2009, que plantean que con objeto de favorecer el desarrollo y disminuir las diferencias interterritoriales las políticas deben ser neutrales desde el punto de vista territorial (spatially blind), la Comisión Europea y muchos de los autores que se mueven en torno a esta (Informe Barca, 2009; Barca y McCann, 2010; Barca, 2011; Landabaso, 2011; Wintjes y Hollanders, 2011; Rodríguez-Pose, 2011), así como la OCDE (véase OECD, 2009; Garcilazo et al., 2010; Garcilazo, 2011), consideran, por el contrario, que las políticas deben tomar en cuenta el contexto (esto es, deben ser spatially aware o place based), contexto este que varía notablemente de unos territorios a otros. En tal sentido, la estrategia de especialización inteligente sería un caso de política place based, dado que para su definición se deben tomar en

\footnotetext{
${ }^{5}$ En los tres casos por él estudiados (a saber, Irlanda, Taiwan e Israel) Breznitz encuentra tres modelos de burocracia claramente diferenciados (en el primero, una burocracia weberiana de estilo inglés, en el segundo una burocracia weberiana tecnocrática y en el tercero una burocracia caóticamente estructurada) que condicionan claramente las estrategias adoptadas por tales territorios.
} 
cuenta cuáles son los activos productivos y bases de conocimiento de la región en las que presenta ventajas comparativas, a partir de las cuales plantear la especialización inteligente (McCann y Ortega-Argilés, 2011; McCann, 2011).

Paralela a esa discusión entre los partidarios de políticas neutrales y de políticas conscientes territorialmente, hay otra discusión entre los partidarios de políticas neutrales sectorial y tecnológicamente (según los cuales, de lo que tendrían que ocuparse las políticas es de acelerar el ritmo de desarrollo o de la innovación, ayudando a superar los fallos de mercado o de sistema que pudieran estar ralentizándolos) y los partidarios de políticas activas sectorial y tecnológicamente (según los cuales, las políticas deben afectar, no sólo al ritmo del desarrollo y de las innovaciones, sino también a su dirección). Pues bien, en este segundo debate las $S^{3}$ se posicionarían en el segundo frente (Foray, 2009a).

Con relación a los dilemas que se le plantean a la estrategia a la hora de elegir el tipo de actividades, en consonancia con los resultados que muestran los estudios empíricos recientes, la especialización inteligente se interpreta no tanto como el impulso a una especialización pura que explote las economías de localización, sino como una "especialización diversificada" (término acuñado por McCann y Ortega-Argilés, 2011) que explote las economías derivadas de la variedad relacionada. En expresión de la Comisión Europea, las regiones deben perseguir una cartera diversificada de actividades relacionadas, encontrando un equilibrio entre especializarse lo suficiente para ser competitivo, pero no demasiado como para dificultar la diversificación y exponer la región a los riesgos de cambios en las condiciones de los mercados o en otros hechos externos e impredecibles (COM(2010) 553 final). Las $\mathrm{S}^{3}$ estarían constituidas por el conjunto de actuaciones emprendidas para avanzar hacia esa diversificación especializada. Camagni (2011) precisa algo más la terminología y prefiere hablar de una estrategia de diversificación y mejora inteligente ("smart diversification and upgrading") de actividades y funciones existentes, esto es, "de una creativa y apropiada diversificación de las especializaciones existentes y de una mejora de su calidad" (p. 59).

Como suele ser habitual, especialmente con corrientes con una vida todavía corta, no hay un consenso pleno sobre qué debe entenderse concretamente por dicho concepto, o de qué plano (sector, clúster, área científico-tecnológica...) hablamos al referirnos al mismo.

\footnotetext{
${ }^{6}$ Gill y el Banco Mundial lo que propugnan es que lo más eficaz para impulsar el desarrollo en todos los lugares es, primero, mejorar las instituciones básicas (servicios sociales y regulaciones de los mercados), de modo que con mejoras institucionales que sean "spatially blinded" se unifiquen los territorios; segundo, que se desarrollen infraestructuras que conecten e integren los territorios; y, tercero, que sólo en circunstancias extraordinarias se recurra a incentivos para atraer empresas a regiones atrasadas. En realidad, de estos tres conjuntos de políticas, sólo la primera sería realmente "spatially blinded" (Gorzelak, 2011; Barca, 2011). Desde los partidarios de las políticas "place based" no se niega que esos tres tipos de políticas sean necesarias; pero se considera que son insuficientes, especialmente para las regiones menos desarrolladas, y que deben ser complementadas por otras políticas que tomen en cuenta el contexto. Respecto a las instituciones "spatially blind" se señala que, si bien la mejora de algunos tipos de instituciones formales (por ejemplo, la del imperio de la ley) puede ser bastante neutral territorialmente, hay todo otro conjunto de instituciones, especialmente de carácter informal, que son plenamente contextuales y cuya necesaria mejora requieren políticas "place based" (Rodríguez-Pose, 2011). Según Barca (2011) lo que realmente distingue los planteamientos del Banco Mundial y de los partidarios de las políticas "place based" es que, además de que los segundos plantean que instituciones e inversiones deben responder a los singulares contextos de cada territorio, el conocimiento de qué hay que hacer debe surgir de la interacción entre fuerzas endógenas y exógenas al territorio (con una gobernanza multinivel), superando las limitaciones de las élites locales para proveer de las instituciones e inversiones necesarias para superar los problemas del desarrollo.
} 
La interpretación que realizan los autores que acuñaron este concepto (Foray et al., 2009; David et al., 2011) es que no se trata de una especialización industrial, sino de una especialización de I+D e innovación. Es más, en los documentos originales de estos autores la especialización territorial de la que se trata es fundamentalmente de la relativa al posicionamiento de las regiones en torno a las llamadas tecnologías de uso genérico (General Purpose Technologies; GPT, en lo sucesivo) ${ }^{7}$. En los documentos de la Comisión Europea y en la propia expresión de lo que ésta entiende por especialización inteligente, en cambio, no parece desprenderse una interpretación tan restrictiva. Y la OECD (2011), insiste en diversos lugares de su informe en no reducir la estrategia de innovación a una estrategia de I+D.

De acuerdo con la visión reduccionista de las $\mathrm{S}^{3}$, a las regiones líder se les recomendaría invertir en la invención de GPT o en la combinación de diferentes GPTs; mientras que a las regiones menos avanzadas, se les propondría invertir en la co-invención de aplicaciones, esto es, en el desarrollo de aplicación de una GPT a uno o varios importantes dominios de la economía regional (Foray et al., 2009). La razón para esa recomendación es que, según los citados autores, las regiones menos avanzadas carecen de los tamaños mínimos y las capacidades para desarrollar invenciones de GPTs, por lo que el gasto que en tales ámbitos desarrollen será ineficiente e ineficaz; mientras que mediante el desarrollo de aplicaciones particulares las regiones menos avanzadas pueden renovar y diversificar sus sectores tradicionales y encontrar nichos en los que sean competitivos globalmente. Son varios los ejemplos que al respecto recogen estos autores: aplicaciones de nanos al sector agroalimentario en Portugal para mejorar el control de calidad en la producción de queso, vino y aceite de oliva; aplicaciones de las bio al sector de recursos marinos en Galicia; aplicaciones de las TIC al mantenimiento de patrimonio histórico y arqueológico en Florencia...

Dejando a un lado el reduccionismo que este planteamiento presenta al centrarse en sólo una de las modalidades de avance hacia la diversificación relacionada (a saber, en las GPT), conviene hacer mención de las reticencias generadas entre ciertos autores (por ejemplo, Pontikakis et al., 2009; Kyriakou, 2009; Giannitsis, 2009), por esa propuesta de que las regiones menos avanzadas se limiten sólo a desarrollar aplicaciones de las GPT a sus actividades, y que por lo tanto renuncien a desarrollar ellas mismas invenciones en las GPT. Estos autores renuentes reconocen, efectivamente, que la especialización presenta ciertas ventajas (aprovechamiento de economías de escala, concentración en las áreas más prometedoras...), pero consideran que de eso se derivan también ciertos riesgos (monopolios, reducciones en variedad, pérdida de flexibilidad...); y asimismo consideran que "las políticas para los seguidores deberían ser flexibles, graduales y evitar el riesgo de impedir u obstaculizar los esfuerzos para construir capacidades y especializaciones en campos prometedores" (Giannitsis, 2009: 5). En el mismo sentido, Etzkowitz y Klofsten (2005) sostienen que una región innovadora precisa múltiples bases de conocimiento para ser capaz de renovarse a sí misma, aunque sólo algunas de ellas vayan a ser aplicables a corto plazo; dado que, si su base académica está demasiado focalizada, no dispondrá de capacidad para

\footnotetext{
${ }^{7}$ La propia OECD (2011) es ese el significado que atribuye a la especialización inteligente: “construir en habilidades existentes en tecnologías genéricas para desarrollar actividades únicas y originales y, a largo plazo, sectores totalmente nuevos" (p. 82). Para McCann y Ortega-Argilés (2011), en ello pudo tener que ver el contexto y preocupaciones originales que llevaron a la creación de esta aproximación, a saber: el retraso que padecía la Unión Europea con respecto a EEUU, por un menor desarrollo y aplicación de una tecnología de uso genérico: las TIC.
} 
desarrollar bases de conocimiento alternativas cuando éste se necesite para su renovación económica ${ }^{8}$. Es más, se aduce que, aunque en apariencia las regiones están llevando a cabo las mismas estrategias (como se derivaría de la frase: "toda región que se precie tiene, en su catálogo, una estrategia bio, una estrategia nano, o una estrategia TIC"), eso sucede porque a niveles agregados no resulta visible la diversificación observable a un nivel de detalle mayor (Giannitsis y Kager, 2009). En el mismo sentido, Smith (2009) sostiene que los datos estadísticos disponibles no permiten sostener si existe demasiada duplicación de esfuerzos o no, y de que no se han manejado datos con suficiente nivel de desagregación para sostener tales valoraciones.

Desde un planteamiento más amplio de la $\mathrm{S}^{3}$, no restringido exclusivamente a las GPT, cabría considerar otras vías de avanzar hacia la diversificación relacionada, o, tal como prefiere denominarla Camagni (2011), hacia una “diversificación y mejora inteligente". Varias de ellas están recogidas en el documento $\operatorname{COM(2010)~} 553$ final, al que anteriormente nos hemos referido ${ }^{9}$. En tal documento se distinguen los siguientes objetivos estratégicos: "retooling", "extending", "emerging" y "cross-sectoral".

- El rediseño ("retooling”) de un sector existente consistiría, según el documento de la Comisión Europea, en el apoyo a la mejora de los recursos humanos y tecnológicos dentro de una industria existente. La Comisión menciona, como casos de rediseño, la aplicación de una GPT a un sector dado (por ejemplo, nanos al sector del papel en Finlandia).

Pero yendo más allá de la aplicación de las GPT al sector, en otro lugar del citado documento la propia Comisión declara que: "los clústeres son un importante componente de las estrategias regionales de especialización inteligente" (p. 5), de modo que la política de clusterización (es decir, del trabajo colectivo en el ámbito de la formación, de la I+D, de la internacionalización... en un conjunto de actividades determinadas) constituiría otra vía de avanzar hacia la especialización inteligente. E incluso, aunque no mencionadas por la Comisión, políticas verticales más tradicionales bien llevadas, como las de reestructuración de sectores, en la medida que fortalecen y mejoran la especialización de un territorio, cabría incluirlas dentro del "retooling".

- La Comisión denomina "emerging" la aparición de una atractiva actividad empresarial por co-emergencia de una actividad de I+D/innovación y una actividad empresarial relacionada. Así, el desarrollo de aplicaciones de TIC para la gestión y mantenimiento del patrimonio arqueológico e histórico -la explotación de sinergias entre esa actividad innovadora y el tradicional cuidado del patrimonio- ha abierto una nueva oportunidad de negocio y ampliado el ámbito de las actividades económicas desempeñadas.

\footnotetext{
${ }^{8}$ El Innovation Union Competitiveness report, 2011 edition, publicado por la propia Comisión Europea, indica al respecto: "En el campo de la investigación y la innovación, es difícil predecir los resultados que se derivarán de las inversiones, y crecientemente, los desarrollos tecnológicos y la innovación pueden estar basados en los resultados científicos de muchas diferentes y a priori no relacionadas disciplinas. El dirigir las decisiones de inversión hacia áreas científicas estrechas puede poner en riesgo la capacidad potencial de desarrollar nuevas tecnologías e innovaciones" (p. 440)

${ }^{9}$ Foray, en una nota elaborada en julio para el mirror group de apoyo a la plataforma $\mathrm{S}^{3}$, menciona cuatro posibles patrones de cambio estructural, que coinciden en gran parte con los recogidos en el documento de la Comisión: modernización de un sector existente, transición a uno nuevo, diversificación por sinergias y fundación radical.
} 
- El "extending" consiste en el descubrimiento de un nicho nuevo, mediante la explotación de economías de alcance. Es decir, las actividades emprendedoras y las infraestructuras educativas y de investigación existentes en un área (por ejemplo, la aeronáutica) se extenderían y aplicarían a una nueva área (por ejemplo, a los GPS y satélites $)^{10}$.

- Por "cross-sectoral" la Comisión entiende la nueva combinación de sectores que ayuda a generar ideas innovadoras para nuevos productos y servicios. En tal sentido, la cooperación trans-sectorial (o a la colaboración inter-clústeres) es uno de los mecanismos para generar ideas para nuevas aplicaciones innovadoras y soluciones integradas típicamente mencionada por la literatura de la variedad relacionada.

Señalemos por último que respecto a los activos que posibilitarían ser competitivos en las actividades económicas en que se especializaría el territorio, aunque en los documentos se hace referencia a la "investigación e innovación" en general (y a los activos complementarios, especialmente de recursos humanos, de aquellos), realmente todos los ejemplos y el espíritu que subyace en los documentos es el de que la innovación se entiende fundamentalmente como innovación tecnológica; e incluso dentro de ésta, la basada en la I+D. Sin embargo, la contabilidad del crecimiento basada en los activos intangibles (Corrado et al., 2005 y 2006; Haskel et al., 2009) ha puesto de manifiesto que los incrementos de productividad de un territorio responden más a otros tipos de factores (mejoras organizativas, diseño...) que al gasto en I+D, y que, en consecuencia, tales factores no deberían ser ignorados a la hora de plantear la estrategia de especialización inteligente de un territorio. Lo anterior resulta más evidente en regiones menos avanzadas, en las que las capacidades de I+D son mucho menores y las ventajas competitivas descansan con frecuencia en otros factores distintos de la $\mathrm{I}+\mathrm{D}^{11}$.

\subsection{Procesos de las estrategias de especialización inteligente}

Foray, padre de la llamada estrategia de especialización inteligente, en sus documentos que han servido de referencia para el impulso de tal tipo de estrategias por la Comisión Europea (Foray y van Ark, 2007; Foray et al., 2009; Foray, 2009a; Foray, 2009b; David et al.,

\footnotetext{
${ }^{10}$ Aunque correspondientes a otras escuelas de pensamiento, los trabajos de Hausmann y Klinger (2006a, 2006b, 2007) e Hidalgo et al. (2007), ofrecen una metodología para mejorar la sofisticación de los productos que exporta un territorio a partir de su actual estructura exportadora. Básicamente dicha metodología consiste en ordenar todos los productos objeto de exportación del mundo en función de su nivel de sofisticación (medida esta, atribuyendo un mayor nivel de sofisticación a los productos exportados por los países con mayor nivel de renta per cápita); en segundo lugar, obtienen una medida de la distancia a que se encuentran unos productos de otros (para eso, recurren a la probabilidad de que un país tenga ventaja comparativa revelada en dos productos para aproximar el grado de proximidad); y, en tercer lugar, atendiendo a los productos que actualmente exporta un territorio, se puede mirar a los productos que, no encontrándose por encima de una distancia dada del mismo, presentan un superior grado de sofisticación y no son producidos por ese país. De esa manera, se pueden diseñar mapas de ruta de avance en la sofisticación de las producciones de un país, a partir de su actual estructura de exportaciones. Como muestran Boschma et al. (2012), este sería un modo de orientar la diversificación de la estructura de un territorio, hacia actividades de mayor valor añadido, basado en el concepto de variedad relacionada.

${ }^{11}$ Véase sobre las limitaciones de las estrategias típicas de especialización inteligente en algunos tipos de regiones, McCann y Ortega-Argilés (2011).
} 
2011), sostiene que el proceso de descubrimiento de en qué actividades debe un territorio especializarse debe ser un proceso emprendedor ("entrepreneurial discovery process"), en el que el papel de los gobiernos no debe consistir en "seleccionar burocráticamente áreas de especialización e impulsar el desarrollo de 'campeones nacionales' en la competición inter-UE" (Foray et al., 2009, p. 4), sino que tal papel debe consistir en:

- proveer de incentivos a los emprendedores y a otras organizaciones (universidades, centros de investigación) para que se impliquen en el descubrimiento de las respectivas especializaciones regionales;

- evaluar y valorar la efectividad de tal apoyo, de modo que tal apoyo no se interrumpa demasiado pronto ni se mantenga demasiado tiempo, y los apoyos se dirijan a sectores económicos con un peso relevante y posibilidades de mejora;

- proporcionar las inversiones complementarias (por ejemplo, en formación y educación) de las especializaciones emergentes;

- proveer de información y facilitar la coordinación y conexiones, tanto entre los diferentes actores del territorio como de éste con otros territorios.

Conviene señalar, no obstante, que en algunos otros documentos el propio Foray acepta un papel para el gobierno que va más allá del mero facilitador y catalizador. Por ejemplo, Foray (2009a) señala: "la cuestión central radica en cómo compatibilizar iniciativas de arriba-abajo (top-down) que favorecen algunos campos (por ejemplo, la respuesta al cambio climático) y la lógica de asignación de recursos impulsada por el mercado, que posibilita 'múltiples experimentos descentralizados'... El principal desafío es, por lo tanto, crear expectativas positivas para múltiples y diversificados agentes con respecto a determinados campos, aunque no tratando de imponer tecnologías predefinidas, enfriando o petrificando la competencia y disipando el extraordinario poder de una economía de libre mercado en impulsar un gran número de experimentos de un modo descentralizado" (pp. 64-65). En otras palabras, "es crucial ser no-neutral al identificar una muy amplia agenda, a la vez que neutral cara a las específicas aplicaciones" (Foray 2009a: 69). En este segundo nivel, el Gobierno no sería quien selecciona la tecnología correcta, sino que, mediante políticas de oferta y de demanda, se limitaría a incrementar la tasa de retorno en los campos elegidos.

Si bien a nivel general el planteamiento de Foray no suscita grandes objeciones, el problema se encuentra en las zonas intermedias. Por ejemplo, dentro de una apuesta por la nanotecnología, ¿no debería el Gobierno, a la hora de plantear la creación de centros de investigación o financiar actividades de los agentes, apostar por las líneas tecnológicas amplias que se adaptan mejor a la estructura productiva local (por ejemplo, en el País Vasco, materiales, antes que fotónica o magnetismo)? ¿Dónde se encuentra la línea que separaría el ámbito macroscópico (en el que estarían justificadas las elecciones del Gobierno) del microscópico (donde serían los agentes los que de modo descentralizado deben realizar sus apuestas)?

Habría que tener en cuenta, por otra parte, que resulta más factible concebir ese mayor protagonismo de los agentes económicos en las regiones más avanzadas que en las menos avanzadas, pues precisamente una de las características de estas últimas es la ausencia o debilidad de los agentes a los que Foray asigna el liderazgo en el proceso de emprendimiento. Ya a comienzos de la década de los sesenta, para explicar los procesos de industrialización de los países europeos de tardío desarrollo, Gerschenkron (1962) había señalado que en los países más atrasados la intervención estatal resultaba más necesaria, tesis que con ligeras 
reformulaciones han sostenido versiones más recientes de las teorías de desarrollo tardío. Y, en el ámbito regional, como señalan McCann y Ortega-Argilés (2011): "Todo lo que sabemos de la Geografía Económica nos dice que los objetivos de la política regional son precisamente aquellos dominios regionales que tienden a carecer de los suficientes niveles (...) de los elementos clave que el esquema de la especialización inteligente requiere para ser una política operativa. Por el contrario, las regiones que aparecen como las más propicias y favorables para operar con procesos del tipo de especialización inteligente son las boyantes regiones centrales". Esto es, debido a la falta de las condiciones necesarias para que en las regiones menos avanzadas se desarrollen apropiadamente los procesos de descubrimiento de la especialización requerida por parte de los emprendedores, en contra de lo que sería la propuesta de Foray, parecería que el impulso de estrategias de especialización inteligente requeriría en tales regiones (que, precisamente, son las más numerosas) una implicación más activa de los poderes públicos.

En los textos de la Comisión Europea referidos a la especialización inteligente, aunque siguiendo a Foray se habla de la necesidad de impulsar "entrepreneurial discovery processes" para identificar esas áreas de especialización, en esos mismos textos no parece excluirse un papel más activo del Gobierno en esos procesos de descubrimiento ${ }^{12}$. Y la OECD (2011) incluso va más lejos, pues, además de señalar que "los gobiernos regionales juegan un rol clave en el reconocimiento de oportunidades para el cambio, en la movilización de recursos hacia la diversificación y en la identificación de nuevas fronteras" (p. 157), indica expresamente que "hay riesgos asociados con un excesiva influencia privada en las estrategias o políticas regionales, que requieren un equilibrio en la combinación de actores y perspectivas públicas y privadas (...) El objetivo es impedir indebidas presiones o incluso la captura de estrategias o instrumentos de financiación particulares" (p. 142) . $^{13}$. Como muestra Breznitz (2007), en algunos casos el sector privado carece de las habilidades y capacidades, o de la disposición, para emprender tales procesos, y el Gobierno deberá asumir un papel activo en dicha identificación y puesta en marcha; sin embargo, a medida que el proceso avanza, el Gobierno debe ir asumiendo un papel más de facilitar y organizar que de ordenar, y de motivar a los otros actores a que se impliquen y asuman el liderazgo del proyecto.

Pero tampoco habría que dar por sentado que esos poderes públicos disponen de las capacidades necesarias para realizar tales apuestas. Como señalan Avnimelech y Teubal (2008), los programas verticales que atienden a un particular sector o tecnología responden a necesidades mucho más difíciles de identificar y su diseño y aplicación son complejos, de

\footnotetext{
12 Por ejemplo, en el Innovation Union Competitiveness report, 2011 edition (p. 440), se incluye a los gobiernos, junto a los emprendedores y universidades, entre los decisores que participan en la elección y desarrollo de una estrategia de especialización inteligente.

${ }^{13}$ En el caso de las empresas, la OECD señala que el riesgo es particularmente elevado cuando una empresa o una especialización sectorial es dominante en la economía regional; y sobre las universidades que tienen una influencia dominante en la estrategia e inversiones públicas, señala que puede llevar éstas lejos de las necesidades del sector privado. Pero asimismo señala la mayor consciencia del sector privado de las tendencias globales y de las condiciones de mercado; y de la universidad y de los centros de investigación, sobre las áreas prometedores para avances en la investigación básica. Por otro lado, tal como antes se ha señalado, para que la captura de rentas por las élites locales no se dé a través de su control del Gobierno regional, Barca (2011) plantea, como principio distintivo de las "place based" estrategias y políticas una gobernanza multinivel, de modo que el factor exógeno imposibilite tales conductas.
} 
modo que requieren una previa acumulación de condiciones, incluida una clara visión. Por eso, en regiones en que esas capacidades no se han desarrollado (como suele ser el caso de las regiones menos avanzadas), tales autores consideran preferible poner el énfasis en los programas horizontales, y, a medida que la experiencia y el aprendizaje de políticas tiene lugar, iniciar el cambio hacia programas más orientados o verticales, con un mayor grado de selectividad.

Los recientes análisis del Regional Innovation Monitor (véase Walendowski et al., 2011) muestran, precisamente, que el proceso de descentralización de competencias (" $d e$ volution") hacia las regiones que se ha vivido en la mayoría de los países europeos, si bien ha dotado a las administraciones regionales de más poderes, no ha ido acompañado por un paralelo crecimiento de las capacidades ("capabilities") de tales administraciones para el ejercicio de tales competencias y el desarrollo de políticas. Salvo en algunas regiones (generalmente, las que de antaño disponían ya de un importante nivel competencial y en las que a través de su ejercicio ha habido un proceso de aprendizaje en el ámbito de las políticas), las políticas puestas en marcha desde el plano regional han presentado generalmente claras deficiencias. Esa falta de capacidad o la captura de las políticas aplicadas por las Administraciones regionales es lo que un elevado número de informes sobre el uso de los fondos de la política de cohesión europea venían precisamente señalando (véanse, por ejemplo, Barca, 2009; Bonnacorsi, 2009; Farole et al., 2009).

En todo caso, lo que debe intentar lograrse es el máximo de participación e implicación de los agentes existentes, y que las decisiones que se adopten, no respondan tanto a la filosofía de políticas del tipo "mission oriented", "enfant industries" o "picking winners", que ignoran las capacidades, existentes o potenciales, del territorio en cuestión (Foray, 2009a; Avnimelech y Teubal, 2008; Porter, 1990), sino que descansen en éstas y estén en consonancia, en la medida de lo posible, con las señales que surjan del mercado. Avnimelech y Teubal propugnan, al respecto, que se aplique un "evolutionary targeting", es decir, que las políticas apalanquen los procesos aparecidos de modo natural y que cumplen una serie de condiciones, porque en tiempos como los actuales en que el ritmo de cambio es tan rápido y complejo, sin tales intervenciones el proceso acumulativo a que darían lugar las fuerzas de mercado sería "demasiado pequeño y demasiado tarde".

A pesar del énfasis que la literatura sobre la $S^{3}$ pone en la importancia del proceso para la definición de las estrategias regionales, el desarrollo que en esta literatura se hace sobre cómo llevar a cabo este tipo de procesos es relativamente escaso y se limita sobre todo a indicar que el proceso tiene que ser un "entrepreneurial discovery processes", sin profundizar en los elementos intangibles necesarios (por ejemplo, el tema del capital social y los tipos de liderazgo) para llevar adelante este tipo de procesos. En lo que sí profundiza más esta literatura es en el papel de los diferentes actores (especialmente, en el del Gobierno) en estos procesos.

\section{RESUMEN Y CONCLUSIONES}

El término estrategia aplicado al territorio resulta cada vez más habitual en la literatura de competitividad e innovación y en el discurso de los decisores públicos, pero sin embargo tal uso no ha venido precedido -salvo en casos excepcionales, como el de Sotarauta 
(2004) - por una discusión expresa sobre qué significa o qué características diferenciales tiene con respecto al uso de término aplicado a otros ámbitos, y en especial a la estrategia empresarial. Ese uso acrítico del término conduce a que, con frecuencia, en los análisis de estrategia territorial, se incurran en ambigüedades y contradicciones, o se ignoren aspectos fundamentales de lo que constituye una estrategia territorial. Este artículo, en su primer apartado, ha tratado de avanzar en esa delimitación del concepto. Siendo un término que, aunque con un origen militar, se ha importado a la literatura económica desde la Dirección Estratégica, en tal apartado se ha revisado brevemente qué significa estrategia en dicho ámbito, con la idea de que dicha reflexión podría ayudar a ordenar la que se haga en el ámbito de la literatura económica y de la innovación. Así se ha creído conveniente distinguir lo que sería el análisis del contenido de la estrategia, del de su proceso. Y a semejanza de la ordenación de preguntas en que cabe ordenar el diseño del contenido de la estrategia empresarial, en el apartado se han especificado los campos a los que el contenido de toda estrategia territorial debiera dar respuesta: en qué clústeres y sectores destacará ese territorio, qué activos específicos ofrecerá para que las empresas en él localizadas sean competitivas globalmente, qué actores se primarán y cuál es el rol de la región con respecto a otros territorios y de los diferentes espacios que la componen.

Pero quizá la diferencia mayor entre la estrategia aplicada a una empresa y la aplicada a un territorio radique en su proceso de formación. Si bien en una empresa, tal proceso puede ser más o menos participativo, pero una vez aprobada la estrategia resulta de aplicación obligatoria, en los territorios, en cambio, los procesos participativos son esenciales, ya que ninguno de los múltiples actores y administraciones dispone de la autoridad, el conocimiento o los recursos para imponer o llevar adelante unilateralmente la estrategia para el territorio por él deseada. La estrategia territorial más efectiva será aquella que combine los aspectos positivos de la aproximación clásica (el análisis consciente y racional, apoyado por ejercicios de prospectiva y escenarios futuros) con el de la aproximación procesual (con espacios para la discusión y negociación que permitan aprendizajes y la generación de visiones compartidas).

La anterior reflexión ofrece una sólida base para abordar el análisis de las estrategias de especialización inteligente $\left(\mathrm{S}^{3}\right)$, idea recientemente lanzada por Foray y una serie de colaboradores, que ha sido asumida e impulsada por la política regional europea. En el análisis de dichas estrategias cabe distinguir, tal como antes se apuntaba, la parte del contenido (el "qué" de la estrategia) de la del proceso (el "cómo" y "por quién"). Respecto al primero, cabría considerar las $\mathrm{S}^{3}$ como una política "place based" (y no, "spatially blind"); y, asimismo, como una política no neutral sectorial o tecnológicamente, sino con un objetivo expreso de incidir también en la dirección del cambio. En particular, se considera que las $\mathrm{S}^{3}$ deberían impulsar una especialización diversificada del territorio.

No hay una única interpretación de cómo avanzar hacia la misma. Desde una visión reduccionista de las $\mathrm{S}^{3}$, ese avance hacia la especialización diversificada se lograría merced a las tecnologías de uso genérico (bios, nano y TIC, fundamentalmente), de modo que unas pocas regiones líderes se especializarían en desarrollar esas tecnologías; y las regiones menos avanzadas, tratarían de desarrollar co-invenciones, para obtener aplicaciones de las mismas a sectores o actividades particulares de su territorio. Ese planteamiento de que las regiones menos avanzadas se especialicen en desarrollar aplicaciones puntuales ligadas directamente a sus sistemas productivos actuales ha sido cuestionado por algunos autores, 
que advierten de los riesgos de una excesiva especialización, especialmente si no se desarrollan capacidades de cambio o "shifting".

En los documentos de la Comisión Europea se exponen otros modos de avanzar hacia la especialización diversificada, no limitados exclusivamente a las tecnologías genéricas, que se denominan por ella "retooling", "extending", "emerging" y "cross-sectoral". En su exposición por nosotros se avanzan divisiones adicionales (distinguiendo, por ejemplo, en el retooling o diversificación dentro de un sector existente, la basada en la aplicación al mismo de una tecnología genérica, de un proceso de clusterización o de una reestructuración sectorial), y sobre todo se destaca que el enfoque de las $\mathrm{S}^{3}$ ha prácticamente ignorado otros tipos de innovación que no están basadas en I+D, y en especial las de carácter no tecnológico, a pesar del mayor impacto que, según la contabilidad del crecimiento, presentan estás en la productividad.

En cuanto al proceso de formación de las $\mathrm{S}^{3}$, el artículo advierte de que el énfasis de Foray en que sea un "entrepreneurial discovery process" y de que el Gobierno no debe participar directamente en las elecciones de carácter microscópico resulta un tanto impreciso. Esa falta de participación directa del Gobierno quizá no resulta aplicable en muchas regiones, en las que el tejido empresarial y las bases de conocimiento son muy débiles, si bien los análisis existentes muestran que no basta con que tales Gobiernos estén dotados de competencias o poderes, sino que también deben de disponer de capacidades; y que en su ausencia, resulta preferible llevar adelante un proceso de "policy learning" basado más en el uso de políticas horizontales. En todo caso, sea cual sea el nivel final de participación del Gobierno, éste debe intentar que el proceso sea lo más participativo posible.

Sin embargo, el nivel de profundización de cómo deben impulsarse los procesos de definición de las $\mathrm{S}^{3}$ es relativamente escaso en relación con la importancia que se da al proceso en esta literatura. En definitiva, el tema de cómo llevar a cabo los procesos de las $\mathrm{S}^{3}$ sigue siendo todavía una caja negra en el que profundizar.

Para terminar, indicar que, por un lado, del análisis conceptual efectuado se desprende que el éxito de las estrategias de especialización inteligente requiere huir de aproximaciones totalitarias, no ligadas a las características del territorio. Por otro lado, el avanzar en definir el cómo llevar a cabo los procesos de definición de estas estrategias requiere ámbitos de experimentación en los que reflexionar y aprender sobre las claves para que estos procesos sean eficientes. Por ambos motivos, es necesario analizar dichas estrategias en contextos regionales concretos, para avanzar hacia conclusiones específicas sobre los factores ligados al éxito de las mismas en dichos territorios, tanto en el "qué" concreto de la estrategia, como en el "cómo" y el carácter más procesual de la misma, y el "por quién".

\section{REFERENCIAS BIBLIOGRÁFICAS}

ARCHIBUGI, D.; HOWELLS, J. y MICHIE, J. (1999). Innovation Systems in a Global Economy. Technology Analysis \& Strategic Management 11 (4): 527-539.

ARUNDEL, A. y HOLLANDERS, H. (2008). Innovation scoreboards: indicators and policy use. En Nauwelaers, C. y Wintjes, R. (eds.) Innovation Policy in Europe (29-52). Cheltenham: Edward Elgar. 
ASHEIM, B. (2009). La política de innovación regional de la próxima generación: cómo combinar los enfoques del impulso por la ciencia y por el usuario en los sistemas regionales de innovación. Ekonomiaz 90: 86-105.

ASHEIM, B. E ISAKSEN, A. (1997). Location agglomeration and innovation towards regional innovation systems in Norway. European Planning Studies 5: 299-330

ASHEIM, B. E ISAKSEN, A. (2002). Regional Innovation Systems: The Integration of Local 'Sticky' and Global 'Ubiquitous' Knowledge. Journal of Technology Transfer 27: 77-86.

ASHEIM, B. y GERTLER, M. (2005). The Geography of Innovation. Regional Innovation Systems. En Fagerberg, J. et al. (eds.) The Oxford Handbook of Innovation. Oxford: Oxford University Press. (pp. 291-317)

ASHEIM, B.; BOSCHMA, R.A.; COOKE, P.; DAHLSTRAND-LINDHOLM, A.; LAREDO P. y PICCAUGA, A. (2006), Constructing regional advantage. Principles, perspectives, policies. DG Research, European Commission

AVNIMELECH, G. y TEUBAL, M. (2008). Evolutionary targeting. Journal of Evolutionary Economics 18: 151-166.

BARCA, F. (2009). An agenda for a Reformed Cohesion Policy. European Commission, Brussels.

BARCA, F. (2011). Alternative Approaches to Development Policy: Intersections and Divergences. En Seminar on Territorial Dimension of Development Policies, papers and proceedings. 18-19 July 2011, Ostróda, Poland. (pp. 45-50). (Descargable en: http://www.mrr.gov.pl/english/Presidency/Main/event_shedule/Documents/Seminar_\%20Papers_and_Proceedings.pdf

BARCA, F. y MCCANN, P. (2010). The Place Based Approach: A Response to Mr. Gill (descargable en http://www.voxeu.org/index.php?q=node/5644)

BATHELT, H. (2003). Geographies of production: growth regimes in spatial perspective (I) - innovation, institutions and social systems. Progress in Human Geography 27 (6): 763-778.

BONACCORSI, A. (2009). Towards better use of conditionality in policies for research and innovation under Structural Funds. The intelligent policy challenge. Report Working Paper of "An Agenda for a reformed Cohesion Policy".

BOSCHMA, R.; MINONDO, A. y NAVARRO, M. (2012). Related variety and regional growth in Spain. Papers in Regional Studies (próxima publicación)

BOSCHMA, R.A. y IAMMARINO, S. (2009). Related Variety, Trade Linkages and Regional Growth, Economic Geography 85 (3): 289-311.

BREZNITZ, D. (2007). Innovation and the State. Political Choice and Strategies for Growth in Israel, Taiwan and Ireland. New Haven \& London: Yale University Press.

CAMAGNI, R. (2011). 'Local knowledge, national vision': challenges and prospects for the EU regional policy. En Seminar on Territorial Dimension of Development Policies, papers and proceedings. 18-19 July 2011, Ostróda, Poland. (pp. 57-63). (Descargable en: http://www.mrr. gov.pl/english/Presidency/Main/event_shedule/Documents/Seminar_\%20Papers_and_Proceedings.pdf

CARLSSON, B. (1994). Technological systems and Economic Performance. En Dodgson, M. y Rothwell, R. (eds.)The handbook of industrial innovation. Aldershot: Edward Elgar. (pp. 13-24)

COOKE, P. (1998). Introduction: origins of the concept. En Braczyk, H.J., Cooke, P. y Heidenreich, M. (1998). Regional Innovation Systems. The role of governances in a globalized world. London: UCL Press. (pp. 2-25).

CORRADO, C.; HULTEN, C. y SICHEL, D. (2005). Measuring Capital and Technology: An Expanded Framework. En Corrado, C., Haltiwanger, J. y Sichel, D. (eds.), Measuring Capital in the New Economy, Studies in Income and Wealth, Chicago: University of Chicago Press.

CORRADO, C.; HULTEN, C. y SICHEL, D. (2006): Intangible Capital and Economic Growth. National Bureau of Economic Research Working Paper 11948m January.

CRISCUOLO, P.; LAURSEN, K.; REICHSTEIN, T. y SALTER, A. (2011). Winning Combinations: Search Strategies and Innovativeness in the UK. Paper presentado en DRUID Conference 2011. 
DALUM, B.; JOHNSON, B. y LUNDVALL, B.-A. (1992). Public policy in the learning society. En Lundvall, B.A. (ed.) National Systems of Innovation. Towards a Theory of Innovation and Interactive Learning. London-New York: Pinter. (pp 296-317)

DAVID, P.; FORAY, D. y HALL, B. (2011). Measuring Smart Specialisation (descargable en: http:// cemi.epfl.ch/files/content/sites/cemi/files/users/178044/public/Measuring\%20smart\%20specialisation.doc)

EDQUIST, C. (1997): Systems of Innovation Approaches- Their Emergence and Characteristics. En Edquist, C. (ed.). Systems of Innovation. Technologies, Institutions and Organizations. London and Washington: Pinter. (pp. 1-35)

ETZKOWITZ, H. y KLOFSTEN, M. (2005). The innovating región: toward a theory of knowledgebased regional development. $R \& D$ Management 35: 243-255.

ETZKOWITZ, H. y LEYDESDORFF, L. (2000). The dynamics of innovation: from National Systems and 'Mode 2' to a Triple Helix of university-industry-government relations. Research Policy 29: 109-123.

EUROPEAN COMMISSION (2010a). Communication from the Commission to the European Parliament, the Council, the European Economic and Social Committee and the Committee of the Regions COM(2010) 553 final. Regional Policy Contributing to smart growth in Europe 2020.

EUROPEAN COMMISSION (2010b). Commission Staff Working Document.SEC (2010) 1183. Document accompanying the Commission Communication on Regional Policy contributing to smart growth in Europe 2020.

EUROPEAN COMMISSION (2011). Innovation Union Competitiveness report. 2011 edition. Luxembourg: Publications Office of the European Union

FAROLE, T.; RODRÍGUEZ-POSE, A. y STORPER, M. (2009). Cohesion Policy in the European Union: Growth, Geography, Institutions. Report working paper for the report "An Agenda for a reformed Cohesion Policy".

FORAY, D. (2009a). Structuring a policy response to a "Grand Challenge". En, Knowledge for Growth. Prospect for Science, Technology and Innovation. Selected papers from Research Commissioner, Janez Potocnik's Expert Group, November 2009.

FORAY, D. (2009b). Understanding “Smart Specialisation”. En Pontikakis, D., Kyriakou, D. y van Bavel, R. (eds.) The Questions of R\&D Specialisation. Perspectives and policy implications. Luxembourg: Office for Oficial Publications of the European Communities.

FORAY, D. y VAN ARK, B. (2007). Smart specialisation in a truly integrated research area is the key to attracting more R\&D to Europe. Knowledge Economist Policy Brief 1, October 2007.

FORAY, D.; DAVID, P.A. y HALL, B. (2009). Smart Specialisation - The Concept. Knowledge Economists Policy Brief 9, June 2009.

FREEMAN, C. (1997). The 'national system of innovation' in historical perpective. En Archibugi, D. y Michie, J. (eds.). Technology, Globalisation and Economic Performance. Cambridge: Cambridge University Press. (pp. 24-49)

FRENKEN, K.; VAN OORT, F.G. y VERBURG, T. (2007) Related variety, unrelated variety and regional economic growth. Regional Studies 41 (5): 685-97.

FRENZ, M. y LAMBERT, R. (2009). Exploring non-technological and mixed modes of innovation across countries. En OECD, Innovation in Firms: a Microeconomic Perspective (pp. 69-110). Paris: OECD publications,

GARCILAZO, J.E.; MARTINS, J.O. y TOMPSON, W. (2010). Why policies may need to be place-based in order to be people-centred (descargable en http://www.voxeu.org/index. php?q=node $\% 2 F 5827$ )

GARMANN JOHNSEN, H. C.; NORMANN, R.; KARLSEN, J. y ENNALS, R. (2008). Democratic innovation -adressing the comprehensive challenges in today's innovation policies: the case of Norway. Paper presented at the conference Regional Innovation Policies, 16-17 October, Santander, Spain. 
GERSCHENKRON, A. (1962). Economic Backwardness in Historical perspective: A Book of Essays. Cambridge: Belknap Press of Harvard University Press.

GIANNITSIS, T. (2009). Technology and Specialisation: Strategies, Options and Risks. Knowledge Economists Policy Brief 8.

GIANNITSIS, T. y KAGER, M. (2009). Technology and Specialization: Dilemmas, Options and Risks?. En Knowledge for Growth. Prospect for Science, Technology and Innovation. Selected papers from Research Commissioner, Janez Potocnik's Expert Group, November 2009.

GILL, I. (2010) Regional development policies: Place-based or people-centred? (descargable en http://www.voxeu.org/index.php?q=node/5644)

GILL, I. (2011). Improving regional development policies. En Seminar on Territorial Dimension of Development Policies, papers and proceedings. 18-19 July 2011, Ostróda, Poland. (pp. 29-34). (Descargable en: http://www.mrr.gov.pl/english/Presidency/Main/event_shedule/Documents/ Seminar_\%20Papers_and_Proceedings.pdf)

GORZELAK, G. (2011). Doctrines of regional policies - their virtues and limitations. En Seminar on Territorial Dimension of Development Policies, papers and proceedings. 18-19 July 2011, Ostróda, Poland. (pp. 15-19). (Descargable en: http://www.mrr.gov.pl/english/Presidency/Main/ event_shedule/Documents/Seminar_\%20Papers_and_Proceedings.pdf

HANSEN, M.T. y BIRKINSHAW, J. (2007), The Innovation Value Chain. Harvard Business Review June, 121-130.

HASKEL, J.; CLAYTON, T,; GOODRIDGE, P.; PESOLE, A.; BARNETT, D.; CHAMBERLIN, G.; JONES, R.; KHAN, K. y TURVEY, A. (2009). Innovation, Knowledge spending and productivity growth in the UK. Interim report for NESTE Innovation Index project.

HAUSMANN, R. y KLINGER, B. (2006a). Structural Transformation and Patterns of Comparative Advantage in the Product Space. CID Working Paper 128.

HAUSMANN, R. y KLINGER, B. (2006b). South Africa's Export Predicament. CID Working Paper 129.

HAUSMANN, R. y KLINGER, B. (2007). The Structure of the Product Space and the Evolution of Comparative Advantage. CID Working Paper 146.

HAX, A.C. y MAJLUF, N.S. (1996). The Strategy Concept and Process. New Jersey: Prentice Hall.

HENNING, M.; MOODYSSON, J. y NILSSON, M. (2010). Innovation and regional transformation. From clusters to new combinations. Malmö (Sweeden): Elanders.

HIDALGO, C.A.; KLINGER, B.; BARABÁSI, A.L. y HAUSMANN, R. (2007). The Product Space Conditions the Development of Nations. Science 317 (5837): 482-487.

ISMERI EUROPA y APPLICA (2010). Distribution of Competences in relation to Regional Development Policies in the Member States of the European Union. Final Report. [Descargable en http://ec.europa.eu/regional_policy/policy/how/additionality_en.htm)

JENSEN, M.B.; JOHNSON, B.; LORENZ, E. y LUNDVALL, B.A. (2007): Forms of knowledge and modes of innovation. Research Policy 36 (5): 680-693.

JESSOP, B. (1998). The Rise of Governance and the Risks of Failure: The Case of Economic Development. International Social Science Journal, March, 29-45.

JOHANNISSON, B. (2008). Regional Development as Entrepreneurial Networking: From Rational Choice to Self-Organizing. En Aranguren, M.J., Iturrioz, C. y Wilson. J. R, (eds.) Networks, Governance and Economic Development. Bridging Disciplinary Frontiers. Edward Elgar.

KAUFMANN, A. y TÖDTLING, F. (2000). Systems of Innovation in Traditional Industrial Regions: The case of Styria in a Comparative Perspective. Regional Studies 34 (1): 29-40.

KYRIAKOU, D. (2009). Introduction. En Pontikakis, D., Kyriakou, D. y van Bavel, R. (2009). The Question of $R \& D$ specialisation. Perspectives and policy implications. Luxembourg: Office for Oficial Publications of the European Communities. (pp. 11-17) 
LANDABASO, M. (2011). Qué políticas pueden o no contribuir al desarrollo regional. En Curbelo J.L., Parrilli, M.D. y Alburquerque, F. (eds.) Territorios innovadores y competitividad. Madrid: Marcial Pons.

LUNDVALL, B-Å. (1992). User-Producer Relationships, National Systems of Innovation and Internationalisation. En Lundvall, B.A. (ed.) National Systems of Innovation. Towards a Theory of Innovation and Interactive Learning. London-New York: Pinter. (pp. 45-67)

LUNDVALL, B-Å. y LORENZ, E. (2010). El Enfoque DUI y de Variedades Relacionadas Innovación y desarrollo de competencias en la economía del aprendizaje. Implicaciones para las políticas de innovación. En Parrilli, M.D. (coord.) Innovación y aprendizaje: lecciones para el diseño de políticas. Zamudio: Innobasque.

MACKINNON, D.; CUMBERS, A. y CHAPMAN, K. (2002). Learning, innovation and regional development: a critical appraisal of recent debates. Progress in Human Geography 26: 293-311.

MAIRESSE, J. y MOHNEN, P. (2007). A survey of innovation surveys: taking stock of a growing literature. Paper presentado en CEPR-Banque de France conference on innovation, Enghienles-Bains, July 6-6.

MCCANN, P. (2011). Notes on the Major Practical Elements of Commencing the Design of an Integrated and Territorial Place-Based Approach to Cohesion Policy. Economic Geography Working Paper June 2011, Faculty of Spatial Sciences, University of Groningen.

MCCANN, P. y ORTEGA-ARGILÉS, R. (2011). Smart Specialisation, Regional Growth and Applications to EU Cohesion Policy. Economic Geography Working Paper 2011, Faculty of Spatial Sciences, University of Groningen.

MINTZBERG, H. (1994). The Rise and Fall of Strategic Planning. Prentice Hall.

MUSCIO, A. (2006). From Regional Innovation Systems to Local Innovation Systems: Evidence from Italian Industrial Districts. European Planning Studies 14 (16): 773-789.

NAHAPIED, J. y GHOSHAL, S. (1998). Social capital, intellectual capital and the organizational advantage. Academy of Management Review 22(2): 242-266.

NAVARRO, M.; GIBAJA, J.J.; FRANCO, S. y MURCIEGO, A. (2011.). El análisis de benchmarking y la identificación de regiones de referencia: aplicación al País Vasco. En Navarro, M. (dir.) Indicadores de innovación y benchmarking. Reflexión y propuesta para el País Vasco. Zamudio: Innobasque.

NEFFKE, F.; HENNING, M., y BOSCHMA, R., (2011). How do Regions Diversify over Time? Industry Relatedness and the Development of New Growth Paths in Regions, Economic Geography, July (próxima publicación)

NELSON, R. (ED.) (1993). National Systems of Innovation: A Comparative Study. Oxford: Oxford University Press

NIOSI, J. (2010). Building National and Regional Innovation Systems. Institutions for Economic Development. Cheltenham: Edward Elgar.

OECD (2009). Regions Matter: Economic Recovery, Innovation and Sustainable Growth. Paris.

OECD (2011). Regions and Innovation Policy, OECD Reviews of Regional Innovation, OECD Publishing.

PONTIKAKIS, D.; CHORAFAKIS, G. y KYRIAKOU, D. (2009). R\&D Specialisation in the EU: From stylised observations to evidence-based policy. En Pontikakis, D., Kyriakou, D. y van Bavel, R. (eds.). The Question of $R \& D$ specialisation. Perspectives and policy implications. Luxembourg: Office for Oficial Publications of the European Communities. (pp. 71-81).

PORTER, M. (1980). Competitive strategy. Nueva York: Free Press.

PORTER, M. (1985). Competitive Advantage. New York: Free Press.

PORTER, M. (1990). The Competitive Advantage of Nations. London y Basingstoke: The Macmillan Press

PORTER, M. (1996) What is Strategy, Harvard Business Review, Nov/Dec 1996. 
PORTER, M. (2010). Microeconomics of Competitiveness. Institute for Competitiveness and Strategy, Harvard.

RODRÍGUEZ-POSE, A. (2011). Spatially-blind strategies as place-based development strategies. En Seminar on Territorial Dimension of Development Policies, papers and proceedings. 18-19 July 2011, Ostróda, Poland. (pp. 85-89). (Descargable en: http://www.mrr.gov.pl/english/Presidency/ Main/event_shedule/Documents/Seminar_\%20Papers_and_Proceedings.pdf

ROSTOW, W.W. (1960). The Stages of Economic Growth: A Non-Communist Manifesto. Cambridge: Cambridge University Press. [Publicado por Fondo de Cultura Económica, México, en 1961]

SACCHETTI, S. y SUGDEN, R. (2003). The Governance of Networking and Economic Power: The Nature and Impact of Subcontracting Networks. Journal of Economic Surveys 17(5): 669-92.

SMITH, K. (2009). Specialisation and Europe's R\&R performance: A note. En Pontikakis, D., Kyriakou, D. y van Bavel, R. (2009). The Question of $R \& D$ specialisation. Perspectives and policy implications. Luxembourg: Office for Oficial Publications of the European Communities. (pp. 41-44)

SOETE, L. (2006), Knowledge, policy and innovation. En Earl, L. y Gault, F. (eds.) National Innnovation Indicators and Policy. Cheltenham: Edward Elgar.

SOTARAUTA, M. (2004). Strategy Development in Learning Cities: From Classical Rhetoric towards Dynamic Capabilities. SENTE-Working Papers 8/2004, University of Tampere.

SUGDEN, R. y WILSON, J. R. (2002). Economic Development in the Shadow of the Consensus: a Strategic Decision-making Approach. Contributions to Political Economy 21: 111-134.

SUGDEN, R. y WILSON, J. R. (2005). Economic Globalisation: Dialectics, Conceptualisation and Choice. Contributions to Political Economy 24: 1-20.

THOMPSON, A.A.; STRICLAND, A.J. y GAMBLE, J.E. (2008). Crafting and Executing Strategy. The Quest for Competitive Advantage (décimosexta edición). McGraw-Hill.

UYARRA, E. (2007). Key dilemmas of regional innovation policies. Innovation 20 (3): 243-261.

UYARRA, E. y FLANAGAN, K. (2009). De sistemas regionales de innovación a regiones como espacios de políticas de innovación. Ekonomiaz 70.

WALENDOWSKI, J.; KROLL, H. y WINTJES, R. y HOLLANDERS, H. (2011). Regional Innovation Monitor. Innovation Patterns and Innovation Policy in European Regions - Trends, Challenges and Perspectives. 2010 Annual Report. Proyecto 0932 para la Comisión Europea.

WHITE, C. (2004). Strategic Management. Palgrave-Macmillan.

WINTJES, R. y HOLLANDERS, H. (2010). The regional impact of technological change in 2020 Synthesis report. European Commission, DG Regional Policy, Brussels.

WINTJES, R. y HOLLANDERS, H. (2011). Innovation pathways and policy challenges at the regional level: smart specialization. UNU-MERIT Working Paper 2011-027.

WORLD BANK (2008). World Development Report 2009: Reshaping Economic Geography. Washingthon, DC. 
